43. U. S. Department of Health, Education and Welfare, Office of Program Analysis. Indicators. Washington, Government Printing Office, 1963. 45 p.

44. Pelton, W. J. Provisions and safeguards of dental service corporation plans. N. J. S. Dent. Soc, J., 30:8-14, July 1959.

45. Pelton, W. J., and Pennell, E. H. Predictability of dental care needs of adults. Am. Dent. A. J., 52:703-8, June 1956.

46. Reed, L. S., and Rice, Dorothy P. Private medical care expenditure and voluntary health insurance, 1948-1961. Social Security Bul., 25:3-13, Dec. 1962.

47. Ryan, K. J. Professional responsibility for the development and guidance of dental service corporations. Am. Dent. A. J., 66:316-21, Mar. 1963.

48. - Complete dental coverage through postpayment and prepayment. Am. Dent. A. J., 68:60-4, Jan. 1964 .

49. Smith, Q. M. Public attitudes toward prepaid dental care. Mich. S. Dent. A. J., 44:119-24, 128, Apr. 1962.

50. Smith, Q. M., and Pennell, E. H. Pricing denticare. Am. Dent. A. J., 69:471-8, Apr. 1960.

51. .... Service requirements in dental prepayment; predictability and adverse selection. Pub. Health Rep., 76:11-8, Jan. 1961.

52. Smith, Q. M., and McDonald, M. J. The dental service corporation; California report. Pub. Health Rep., 77:919-27, Nov. 1962.

53. Source book of health insurance data. New York, Health Insurance Institute, $1961.80 \mathrm{p}$.

54. Three year study of the first dental health care plan. Am. Dent. A. J., 65:550-1, Oct. 1962 .

55. U. S. Bureau of Census. City and county data book. Washington, Government Printing Office, 1962. XXIV + 669 p. (p. 2, 33)

56. U. S. Department of Commerce. Personal income and outlay. Survey of current business, 42:14, July 1962.

57. Williams, C. H. Subversion is not confined to politics. Calif. S. Dent. A. J., 39:99-100, 130. Apr. 1963.

58. Williams, M. W. The insurance company in prepaid dental programs. Ohio S. Dent. Soc. T., 36:94, Tune 1962 .

59. Wisan, I. M. Provision of dental care in community dental programs. J. Dent. Child., 26:233-7, 3rd Quar. 1959.

60. Young, W. O. Dental health. n. 5-94. (In Hollinshead, B. S., Director. Survev of dentistry; the final report. Washington, American Council Education, 1961. XXIV +608 p.)

\title{
THE DENTISTS AND THE SERVICES THEY PROVIDED FOR TWO POPULATIONS; COMMENTS ON METHODOLOGY OF STUDY
}

\author{
By Harry L. Draker, D.D.S., M.P.H.," Charles A. Metzner, Ph.D. ${ }^{* *}$ \\ and Norman C. Allaway, M.S.***
}

\section{EASYP-Dr. Harry L. Draker points out the pitfalls in an interview- survey and confirmation by dentists' records.}

A dilemma must be faced in studying the health of populations because data on health are thought to be more valid and reliable when obtained as professional judgments, a conclusion that should be true for diagnoses and other technical information. It has been found difficult, under the system of providing

fPresented at the 91st Annual Meeting of the American Public Health Association, Kansas City, Missouri, November 11-15, 1963; a study supported in part by Grant DH 00013-03 from the National Institutes of Health.

${ }^{*}$ Evaluation Consultant, Public Health Research, Development and Evaluation Group, N.Y.S.

Department of Health, Albany, N. Y.

* Professor of Public Health Economics, University of Michigan, Ann Arbor, Michigan.

* Principal Biostatistician, N.Y.S. Department of Mental Hygiene, Albany, N. Y. (formerly with N.Y.S. Department of Health). 
services for health in the United States to associate professional persons with defined populations, and in particular with such designated items as income, education and other characteristics of status which can only be obtained from the persons concerned.

Studies which utilize populations obtained from the records of dental offices suffer from the bias of self-selection because only those who have received care are recorded, and those who never or rarely receive care are missed entirely.

Studies in hospitals always have been plagued with this problem ${ }^{2}$ and, even when an entire state is studied, difficulties develop along the boundaries." Such use of large units was judged to be insufficient for gaining information from institutional data even for the U. S. National Health Survey, ${ }^{3}$ but a number of validating studies have been completed to determine how closely data on a population relate to professional measures and judgments. ${ }^{*}$

The study to be described attempted to resolve the issue by "grasping both horns" of the dilemma. Data were obtained both from populations and profession. Two populations were interviewed concerning their utilization of dental services, their expenditures and their attitudes. The names of the dentists providing the services were obtained, and these dentisis subsequently interviewed. The populations were those of Erie and Livingston Counties in the State of New York. This study was developed as a pilot-project, and the two counties were chosen because Erie County, containing Buffalo, New York, is predominantly urban and Livingston County, predominantly rural (Table No. 1).

Study of Family Expenditures for Dental Care in New York State (IV) SUMMARY OF ECONOMIC CHARACTERISTICS, BY COUNTIES (1960 Census Data)

Table No. 1

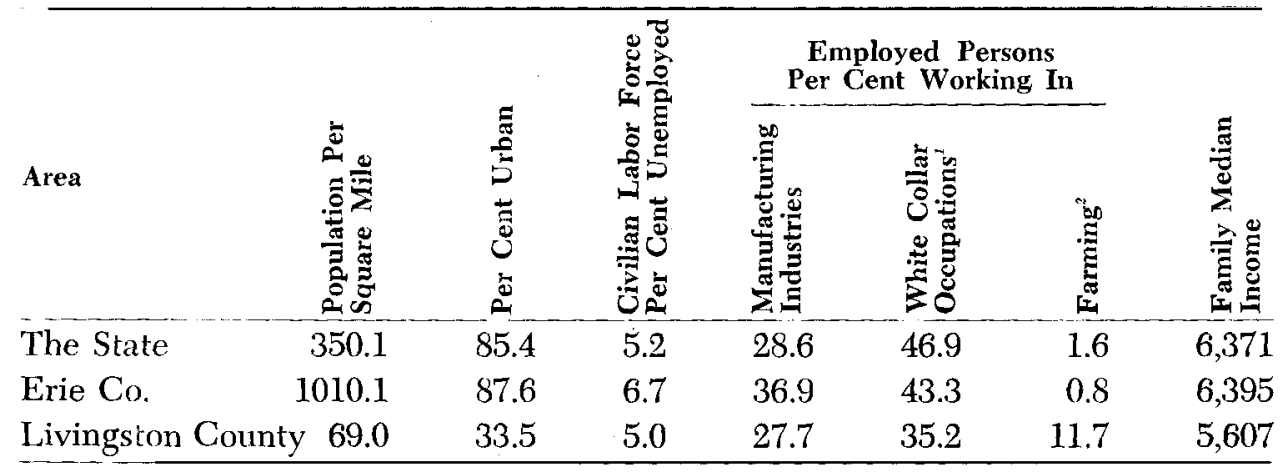

1. Professional, managerial (except farm,) clerical, and sales.

$\because$ Farmers, farm managers, farm laborers and farm foremen.

An additional factor in the choice of these particular urban and rural counties was the existence of recent sampling frames, which were obtained from Dr. J. E. Dowd of Roswell Park Memorial Institute, Buffalo and Professor R. A. Danley of the Department of Rural Sociology, Cornell University. From these frames, probability samples of private, noninstitutional households were developed by the selection of standard areas. 


\section{Design of Study}

A schematic illustration of the design of the study will be found in Figure A. The complexity of the interrelationship of respondents and dentists was apparent. These complexities were fully observable in the subsequent assembly and analysis of the data.

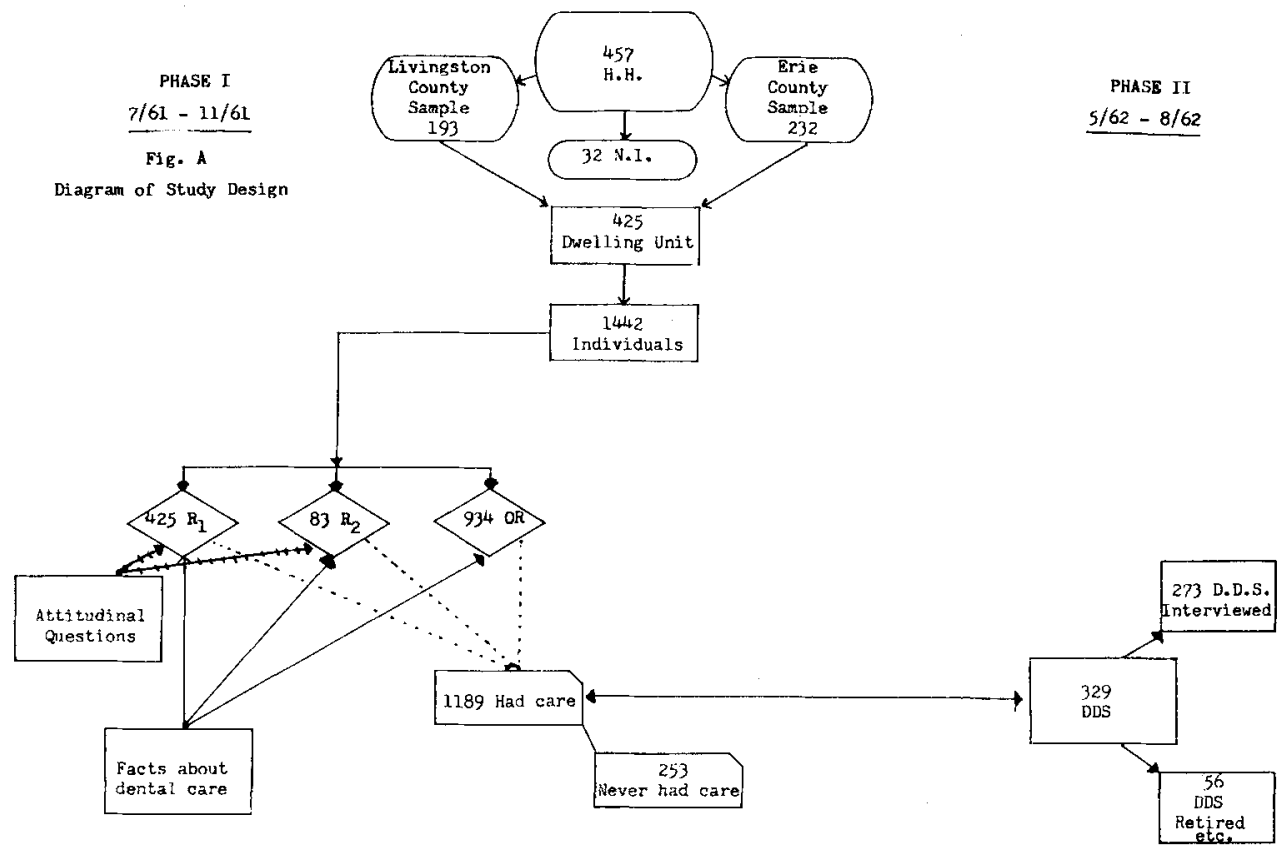

\section{Sampling}

The frame for the population of Erie County included urban and suburban Buffalo, with a combined population in 1960 of 869,826 persons. Census-tracts served as strata in the suburban areas outside of the city of Buffalo. These suburban areas were distributed among the census-tracts proportionately to the number of households in the tract. Those tracts having less than the necessary number of households to ensure at least one household being drawn, were merged with an adjoining tract (of the same socio-economic quartile). Every household in the suburban areas, hence was associated with some censustract in which there was a nonzero chance of the household being selected.

No satisfactory directory existed for suburban areas, therefore, the materials of the Enumeration District of the U. S. Census Bureau of 1950 were used. Enumeration-districts were selected with a probability proportional to their 1950 population, and with replacements. Selected districts then were cruised quickly and subdivided further into segments of approximately 80-100 households. Segments were selected with equal probability, with replacement. The selected segments then were cruised, and a complete listing of households was made. From this listing, households were selected with equal probability 
and without replacement. The listings of households within selected segments (very often a single block in the highly populated suburbs) provided the listings from which the sample of households was selected.

Within the city of Buffalo, a simple randomized sample of households was selected from the 1960 Directory of the City of Buffalo. No stratification was used because of the enormous amount of labor incrossindexing a listing of households to get the proper advance selection from each census-tract. The resulting Erie subsample available for the study contained 766 individuals.

The population of Livingston County in 1960 was 44,053. Localities of 2,500 or more residents (and, therefore, non-rural by the definition of the U. S. Bureau of Census) were excluded from the sample, so that a population of 29,287 constituted the rural universe.

A county map was divided into numbered, geographically identifiable segments. An initial segment was selected randomly, and subsequently other segments were established by methods of randomization. The final sample of rural households contained 676 individuals.

Of 250 dwelling-units in Erie County, 18, or 7.1 percent did not result in interviews. In the sample for Livingston County 14, or 7.6 percent of 207 households were vacant, could not be located or interviews were refused. The overall rate for noninterviews, therefore, was about 7.0 percent for the study as a whole, or even somewhat less when vacancies were excluded.

\section{Respondents}

In both counties, one adult in every household was designated as a primary respondent $\left(R_{1}\right)$. When the actual head of a household could not be interviewed (father at work, for example), the mother or oldest adult child present was so designated. Either then spoke for all members of the household, except for those with independent incomes; those enumerated and interviewed for data on dental care only were grouped as "other respondents," (ORx). Secondary respondents $\left(R_{\mathcal{2}}\right)$ were adult members of the household, related or not, who had independent incomes.

The questionnaire directed attitudinal questions at $R_{1}$ and $R_{2}$ only, but data on dental care were obtained for all members of the household, wherever possible. Members of the household who never had dental care were so identified because this group appeared to be of particular interest. Data on this group have not been reported. Altogether, 1442 interviews were obtained from 425 households. Summary data are presented in Table No. 2.

Interviewing was carried out from July, 1961, to November, 1961, for Phase 1, the study of population, and from May, 1962, to August, 1962, for Phase 11, the study of the dentists for which, of course, a separate questionnaire had to be developed.

The 1442 persons who were interviewed designated 329 dentists as those from whom they had received their last dental treatment. In many instances, dentists did not reside in the county of the patient's residence. Figure B shows the pattern in Livingston County. This observation documents not only the mobility of the rural population but also a regionalization consistent with the concept of "natural marketing areas," which apparently exceed metropolitan marketing centers. Fifty-six of the dentists had retired, died, or were otherwise not locatable. 


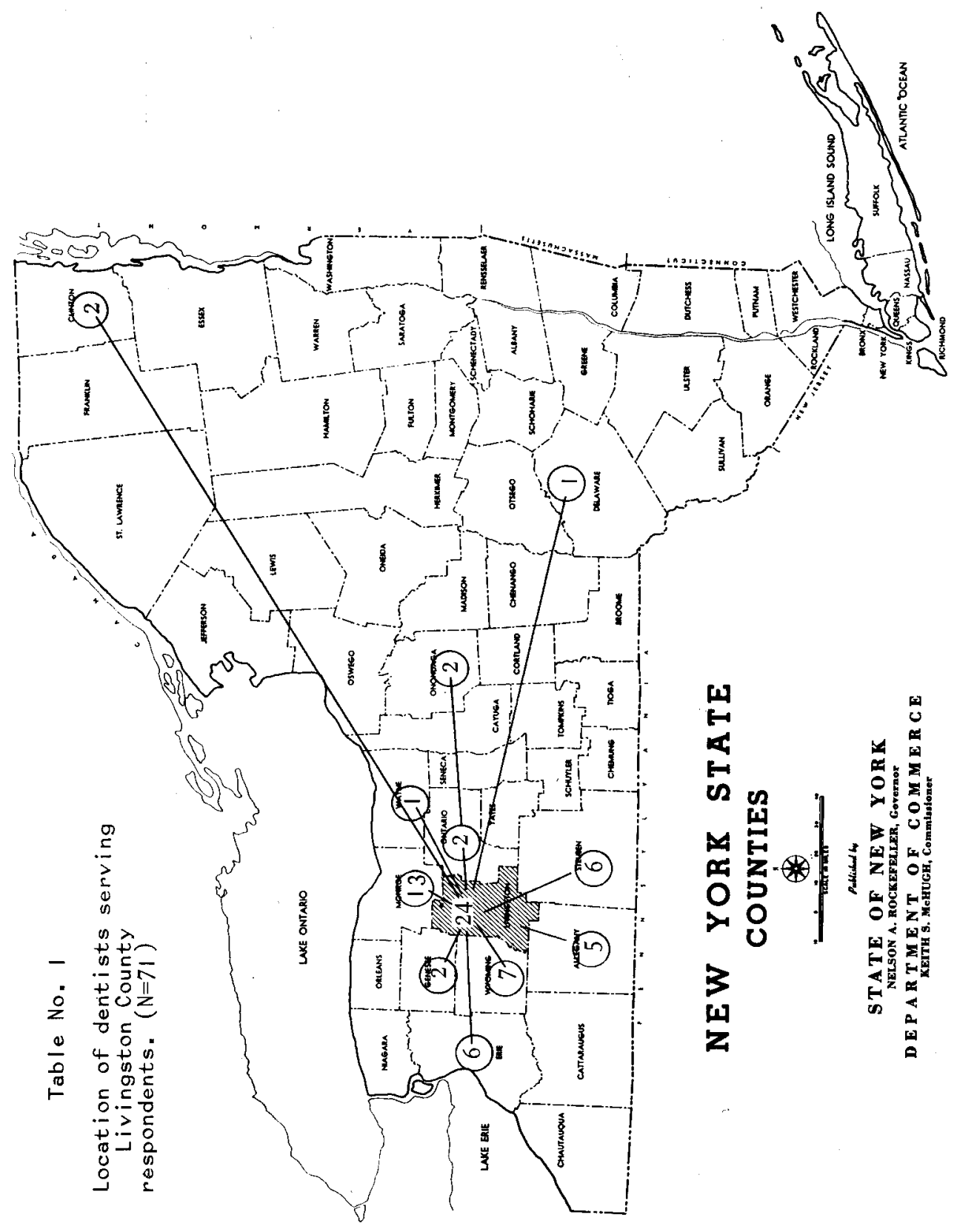


Study of Family Expenditures for Dental Care in New York State (IV)

SUMMARY DATA

Table No. 2

\section{PHASE I}

1. Households in sampling plan

2. Non-Interviews: Refusals, died, house vacant or unable to locate

3. Households studied

4. Individuals studied

5. Primary respondents

6. All other respondents

7. Persons who had dental care

8. Persons who never had dental care

\section{PHASE II}

9. Dentists identified in sample

10. Dentists retired, died, unable to locate

\begin{tabular}{ccc} 
Livingston & Erie \\
Total & Co. & Co. \\
\hline
\end{tabular}

11. Total dentists interviewed

\begin{tabular}{rrr}
32 & 14 & 18 \\
& $=6.8 \%$ & $=7.2 \%$ \\
425 & 193 & 232 \\
1,442 & 676 & 766 \\
425 & 193 & 232 \\
1,017 & 483 & 534 \\
1,189 & 552 & 637 \\
253 & 124 & 129 \\
\hline & & \\
329 & 71 & 258 \\
56 & 21 & 35 \\
273 & 50 & 223
\end{tabular}

\section{Validating Data}

1. Mutual Recognition of the Relationship of Patient and Dentist

Table No. 3 presents data on mutual identification of patients and dentists. It will be recalled that the names of the dentists were obtained from the respondents during interviews.

Study of Family Expenditures for Dental Care in New York State (IV) MUTUAL RECOGNITION OF PATIENTS AND DENTISTS

Table No. 3

\begin{tabular}{ccc}
\hline Number of responses leading to identifiable dentist & $\begin{array}{c}\text { Number Of } \\
\text { Records } \\
1,145\end{array}$ & $\begin{array}{c}\text { Per Cent Oे } \\
\text { Total } \\
\text { Records } \\
100.0\end{array}$ \\
\hline Case verified by D.D.S. & 815 & 71.2 \\
Records actually found & 782 & 68.3 \\
Patient recognized but no record available & 33 & 2.9 \\
Patient not recognized by D.D.S. & 330 & 28.8 \\
\hline
\end{tabular}

One thousand one hundred and eighty-nine patients reported care, and several were known to have used more than one dentist. The expected number of dental records should, therefore, have exceeded 1189. Dentists were identi- 
fied, however, only in connection with 1145 episodes of treatment of this potential universe, the dentists could acknowledge but 815 , or 71 percent.

In determining a base for the comparison of data on patient and dentist, the dentist was accepted as the fixed point when he produced a record. The dentist was able to do so for 96 percent of the 815 patients. More patients identified themselves with specific dentists than the records indicated.

This variability might have been produced by several causes. There could have been deficiencies in the dentist's records; errors in memory on the part of the respondent could be responsible; and there could have been errors in the "hearsay knowledge" of the respondents who spoke for other members of the household. This problem could have been complicated further because some respondents were patients of more than one dentist, and some families divided their care between as many as four dentists.

\section{Date of Recall for Care}

In determining the accuracy of respondents' statements regarding the date of care, it was found that respondents tended to overestimate the lapse of time. Only 26 percent of 622 patients were accurate in recalling the correct year of their dental care (Table No. 4).

\section{Study of Family Expenditures for Dental Care in New York State (IV) VERIFICATION OF TREATMENT DATE: DENTIST"S RECORD VS. PATIENT'S STATEMENT}

Table No. 4

Cases with date of care given by both patient and record 622

Date recalled within one year 160

Percent of Total group

Date recalled within 3 years

Percent of total group 59.6

Date recalled within 5 years 463

Percent of total group

The differential in time between the date on the dentist's record and the date obtained by the interview was within plus or minus 3 years for about 60 percent of all who had care and whose records were available. Obviously then, the recall of the date was not reliable, and there were indications that a respondent's attitude toward dental services may have had a bearing on his ability to recall.

\section{Identification of Level of Income}

The system of rendering dental care in the United States depends for its success to a substantial degree on the proper and equitable application of a sliding scale of fees. The comparison of levels of income as estimated by the dentist with the levels of income obtained from interviewing households were, therefore, interesting. (Table No. 5) 
Study of Family Expenditures for Dental Care in New York State (IV)

DENTIST'S ESTIMATE OF PATIENT'S

INCOME VS. TRUE INCOME LEVEL

Table No. 5

\begin{tabular}{lcccc}
\hline \hline $\begin{array}{l}\text { Patient's } \\
\text { Economic } \\
\text { Level }\end{array}$ & \multicolumn{4}{c}{ Dentist's Estimates } \\
& Total & Low & Medium & High \\
\hline Low & 245 & 37 & 198 & 10 \\
\cline { 2 - 5 } Medium & 51 & 9 & 42 & -- \\
High & 169 & 27 & 139 & 3 \\
\hline & 25 & 1 & 17 & 7 \\
\hline & $\%$ agreement: $\frac{155}{245}=41 \%$ & &
\end{tabular}

The actual data on income were obtained from 74 percent of the respondents. Because some dentists were unable or unwilling to estimate the level of income of their patients, comparison was possible for only 65 percent. The estimates appear in the table. It will be seen that dentists estimated income correctly in 41 percent of the instances (total 155). There was some tendency to relegate medium to low, and high to medium, but out of a total of 51 patients with low incomes, 42 ( 82 percent) were classified in the next higher class of income. The acuity of dentists' judgment of economic level was depreciated further when one keeps in mind that the "medium" group in itself was a rather poorly defined "catch-all."

\section{Agreement on Types of Services; Patients' Statements vs. Dentists' Records}

One might be inclined to expect that patients would be capable of recalling the services that were performed for them. This assumption was not justified by data obtained in this study even when somewhat dramatic services were performed (e.g., extractions.) The best agreement, in fact, between the data of patients and dentists was exhibited for fillings (about 66 percent) followed by cleanings (about 50 percent). Even this level of agreement lost most of its impact because these two services are performed more frequently than others, and attest to the truism, "the most frequent diseases occur most frequently."

"Extractions" were recalled correctly about as often as "surgery" (better than 40 percent). The records of dentists regarding dentures of all types verified that this service could be recalled correctly in somewhat less than 40 percent of instances.

\section{Review of Comparability}

It should be remembered, in viewing Tables No. 3 through No. 6, that they were based on those instances in which the patient-dentist relationship was mutually recognized. The total error was governed by the amount of error in Table No. 3. The table does not reflect those instances in which a dentist may 
have given information regarding a service performed which the patient did not report-an aspect of validation which was not examined.

Some of the difficulties encountered were reflected in the tables, but not explicitly. While a design of study that permitted comparisons between the statements of patients and the dentist's records has many attractions, it also has inherent limitations. The quality of dentists' records is one of the variables which cannot be controlled. Comparability is limited further, though not made impossible, by differences in terminology and problems of semantics. This problem in comparability became especially clear when asking about "work completed." This term meant, at varying times, either completion of all needed work at that time, all planned and agreed work, and a particular course of treatment, since followed by other courses. Clarification of the questionnaire in this feature could reduce a source of discrepancy. Other information may result from a mere projection of attitude, e.g., date that the care was received. Some estimates, on the other hand, were obtained from dentists on items for which the patient should have been the valid source, income for example. This correlation suffers from the demonstrated inability of the dentist to assess the income of patients correctly.

\section{Summary}

The problems of securing authoritative professional data concerning dental services which are, at the same time, related to reliable characteristics of a population was attempted in this pilot-study of dental care by interviews with samples of an urban and an rural New York county, and with the dentists from whom they reported receiving this care.

\section{Study of Family Expenditures for Dental Care in New York State (IV) AGREEMENT BETWEFN TYPES OF SERVICES (PATIENTS' VS. DENTISTS' RECORDS)}

Table No. 6

\begin{tabular}{|c|c|c|c|c|c|c|c|c|c|}
\hline & 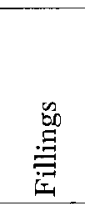 & 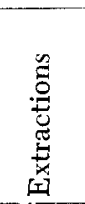 & 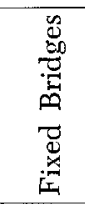 & 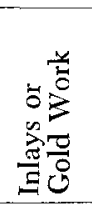 & 莺 & 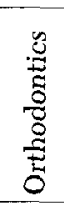 & 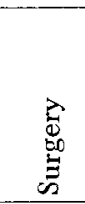 & 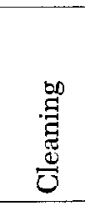 & $\frac{\tilde{g}}{\tilde{0}}$ \\
\hline $\begin{array}{c}\text { Number of Patients with } \\
\text { Dentists' Records }\end{array}$ & 587 & 446 & 14 & 4 & 258 & 11 & 7 & 374 & 86 \\
\hline $\begin{array}{l}\text { Number of Dentists' } \\
\text { Agreeing with } \\
\text { Patient's Statement }\end{array}$ & 390 & 197 & 3 & 0 & 102 & 0 & 3 & 189 & 34 \\
\hline Percent Agreement & 66.4 & 44.2 & 21.4 & $\overline{0.0}$ & 39.5 & 0.0 & 42.9 & 50.5 & 39.5 \\
\hline
\end{tabular}

Procedures have been discussed, and data from both patients and dentists presented relative to (1) mutual recognition of the patient-dentist relation, (2) recall of date of care; (3) income of patient, and (4) the type of service provided.

The value of the dual approach was verified by the low agreement between dentist and patient both on items for which the dentist may be considered a primary source and those for which the patient may be considered primary. Ob- 
taining information from one only entails heavy losses on items for which the other is primary.

\title{
Bibliography
}

1. Belloc, Nedra B. Validation of morbidity survey data by comparison with hospital records. Am. Statistics A. J., 49:832-6, Dec. 1954.

2. Winter, K. E., and Metzner, C. A. Institutional care for the long-term patient. Research Series No. 7, Ann Arbor, University of Michigan, Bureau of Public Health Economics, 1958. VI $+137 \mathrm{p}$.

3. U. S. National Center for Health Statistics. Origin, program and operation of the U. S. National Health Survey. Series 1, No. 1, Washington. Government Printing Office, 1963. 41 p. (p. 2-3)

4. - . A study of special purpose medical techniques. Series D, No. 1, Washington, Government Printing Office, 1960. 27 p.

\section{TESTING FOR THE EFFICACY OF A MOUTHWASH*}

\author{
Hyman K. Schonfeld, D.D.S., Dr. P.H. *
}

Read this step-by-step guidance on organizing a project of research to test the benefits of a mouthwash.

As members of a health profession it is a professional function, or obligation and duty to prevent disease and disability. If disease cannot be prevented, it must be cured or at least modified. Through the application of knowledge, the health professions are committed to getting all people to operate at their maximum efficiency with a distinct feeling of well being. This state of utopia, while perhaps always just beyond grasp, must be sought constantly. Among the weapons to be used are drugs and the cosmetic agents which include mouthwashes.

The term mouthwash, as with almost any product, can mean different things to different individuals. The American Dental Association, in its edition of Accepted Dental Remedies for 1964, states: "A mouthwash is generally regarded as a medicated liquid used for cleansing the mouth or treating diseased states of the oral mucous membrane."

Some people use a specific mouthwash, under the supervision of a dentist or a physician, to aid in the treatment of a disease or its symptom. Other people expect the mouthwash to cleanse the mouth by removing food particles. Some consumers hope for greater social acceptance through the elimination, reduction or masking of odors in the mouth often associated with specific foods or liquids. Still other users appear to face each day with a feeling of well being and with confidence after using a stimulating and refreshing mouthwash.

The specific expectation is a result of many factors, some known only to the consumer himself. Each person's expectations are a reflection of his values, attitudes and beliefs. These expectations, however, are not solely the concern of the consumer himself. They are equally the concern of the investigator,

\footnotetext{
* Paper presented July 15, 1964, at the Bi-Regional Conference of State and Territorial Dental Directors, Regions IV and VII, Santa Fe, New Mexico.

* Clinical Research Services, Warner-Lambert Research Institute, Morris Plains, New Jersey,
} 